\title{
INFOLYN - ELEVANDO A SATISFAÇÃO DOS CLIENTES INTERNOS E EXTERNOS
}

$\begin{array}{ll}\text { Recebido em: } & 21.12 .2017 \\ \text { Aprovado em: } & 22.12 .2017\end{array}$

\section{Adilson Aderito da Silva}

Doutor em Administração de Empresas (2009) e Mestre em Educação, Arte e História da Cultura (2000) pela Universidade Presbiteriana Mackenzie. Graduado em Matemática pela Fundação Santo André (1993). Professor do núcleo docente permanente do Mestrado Profissional em Administração do Desenvolvimento de Negócios da Universidade Presbiteriana Mackenzie - UPM e atual diretor acadêmico do Centro de Ciências Sociais e Aplicadas. Desenvolve projetos de pesquisa na área de Administração Estratégica com foco em Governança de Negócios, Incerteza no Ambiente Organizacional, Ambiente Competitivo e Alianças Estratégicas Contratuais e Custos de Transação. Tem experiência com Tratamento e Modelagem de Dados por Equações Estruturais e Técnicas Estatísticas Multivariadas. Email:asilva@mackenzie.br

\section{Alberto de Medeiros Júnior}

Professor do Mestrado Profissional em Administração do Desenvolvimento de Negócios da Universidade Presbiteriana Mackenzie. Coordenador do Projeto de Pesquisa LabMIN - Laboratório Mackenzie de Inteligência de Negócios. Coordenador do Programa de Extensão MackEmpresas. 


\section{Andrea Cristina Deis Rodrigues}

Master Coach pela SBC Coaching, com certificação pelo BCI - Behavioral Coaching Institute e ICC - International Coaching Council. É licenciada em Pedagogia pela Unifai e pósgraduada em Gestão Empresarial pela FGV-SP.

E-mail:andrea@andreadeis.com.br

\section{Elisabete dos Santos Fernandes}

Consultora de negócios no Sebrae/SP, formada em Administração, especialista em desenvolvimento e gestão de modelos de negócios inovadores, gestão de equipes e formação de líderes, professora universitária e palestrante. E-mail: elisabeteesf@hotmail.com

\section{Lucas Augusto Perez dos Santos}

Advogado, especialista em Direito Empresarial pela FGV-Direito-SP, com passagem em empresas nacionais e multinacionais gerenciando áreas de Compliance e Auditoria Interna. E-mail: lucasaugusto.ps@hotmail.com

\section{Mariana Pires Dias}

Gerente de divisão da Diretoria de Distribuição Sudeste do Banco do Brasil, é pós-graduada em Administração pela FGV e em Gestão de Operações e Serviços pelo Inepad/UnB. E-mail:marianapiresdias80@gmail.com

\section{RESUMO}

A empresa InfoLyn desenvolve e implementa softwares voltados ao mercado de varejo em todo o território nacional. À luz dos pressupostos e dimensões da Teoria dos Custos de Transação (TCT), neste relato são apresentados os resultados das observações que culminaram no mapeamento das principais fontes de custos que emergem na área de Suporte Técnico da empresa. Identificou-se que a assimetria de informações na InfoLyn é potencializada pela racionalidade limitada dos agentes e dá margem ao comportamento oportunista, problemas de gerenciamento da equipe, atendimento da base de clientes e, consequentemente, custos de transação. Observou-se ainda que existe dificuldade para utilização dos sistemas pelos usuários e que o elevado número de customizações necessárias às operações da empresa onera a área de Suporte Técnico. Como resultado prático do estudo propôs-se à empresa alternativas para melhoria da área embasadas em processamentos de informações das bases de dados do suporte técnico em software de tomada de decisão por dashboard. Essas alternativas permitirão à InfoLyn atuar como protagonista no relacionamento com os clientes, atrair novos contratos e diminuir os custos de transação e de operação identificados.

\section{PALAVRAS-CHAVE}

Custos de transação. Suporte técnico. Apoio à Decisão. Integração. 


\section{INTRODUÇÃO}

A InfoLyn Sistemas é uma empresa de desenvolvimento e implementação de softwares voltados ao mercado de varejo. Identificou-se na InfoLyn um problema relacionado à racionalidade limitada, em que os clientes internos e externos apresentam baixa capacidade de interpretação e processamento das informações disponíveis que dão margem ao comportamento oportunista refletido por disputas de poder e busca de interesses próprios entre os parceiros.

Diariamente a empresa depara com a necessidade de gerenciar situações de incerteza, geradas pela assimetria de informações entre as partes, tanto interna quanto externamente. Nota-se que a necessidade do cliente difere daquilo que a área técnica interpreta e desenvolve, assim como a manutenção da operação pelo suporte técnico daquilo que efetivamente foi vendido.

A partir do panorama e diagnóstico das operações da empresa foi perceptível a necessidade de implementar algumas mudanças na InfoLyn, notadamente com relação à precificação de seus contratos; melhoria do processo de comunicação externa e do relacionamento interpessoal dos sócios e funcionários; revisão de métodos; melhorias nos índices de desempenho e satisfação dos clientes, cujo relacionamento cotidiano se dá por meio do suporte técnico.

Análises das informações e propostas de ação para a diminuição dos custos de transação relacionados à precificação de contratos e medidas para aprimoramento de questões comportamentais serão abordadas no presente estudo. Portanto, o objetivo deste relato é apresentar as alternativas gerenciais obtidas no processamento das informações disponíveis do setor de Suporte Técnico por meio de um Sistema de Apoio à Decisão em dashboard, para aprimorar a área de Suporte Técnico e minimizar os custos de transação na InfoLyn.

\section{CONTEXTO E REALIDADE INVESTIGADA}

A InfoLyn foi fundada em 2008 e está localizada em cidade do interior próxima à capital do estado de São Paulo. Atua em todo o território nacional e conta com 30 colaboradores e quatro sócios distribuídos entre as áreas administrativa, comercial, desenvolvimento e suporte técnico. Sua especialidade é avaliar e reconhecer as necessidades de seus clientes e propor soluções 
tecnológicas que visam minimizar custos e gerar valor aos clientes.

A missão declarada da empresa é ser um gerador de valor para seu negócio, oferecendo serviços e soluções tecnológicas voltadas para empresas de diversos segmentos e tamanhos, trazendo a satisfação dos clientes e parceiros, para garantir a qualidade do relacionamento no longo prazo.

Os principais clientes da InfoLyn são distribuidores varejistas de diversos setores que possuem rede de vendedores que necessitam emitir nota fiscal no ambiente externo da empresa. Os ramos de atividades que mais utilizam esse tipo de serviço são: distribuidores multimarcas, distribuidores exclusivos, indústrias e distribuidores de produtos higiênicos.

Segundo a Associação Brasileira das Empresas de Software, o mercado de Tecnologia de Informação (TI) no Brasil, incluindo hardware, software e serviços, aumentou 9,2\% no ano de 2015, contra os $5,6 \%$ da média global de crescimento. No mundo, os investimentos nesse setor somaram US\$2,2 trilhões (ABES, 2016).

Já no ranking de investimento no setor de TI na América Latina, o país se manteve em $1^{\circ}$ lugar, com $45 \%$ dos investimentos, somando US\$ 59,9 bilhões, seguido por México
(20\%) e Colômbia (8\%). Ao todo, a região latino-americana soma US\$133 bilhões. Responsável por quase metade dos investimentos em TI na região, o Brasil mantém uma liderança regional relevante (ABES, 2016).

A InfoLyn pode ser considerada uma empresa de pequeno porte no setor em que atua, com faturamento anual de aproximadamente R \$ 2,5 milhões. Entre seus principais concorrentes destacam-se empresas de desenvolvimento de software, gestão empresarial, soluções digitais e dispositivos móveis.

\section{DIAGNÓSTICO DO PROBLEMA}

A InfoLyn depara-se hodiernamente com a necessidade de gerenciar situações de incerteza provocadas por assimetrias de informações entre as partes, assimetria essa potencializada pela racionalidade limitada e pela falta de clareza na comunicação entre os agentes.

Durante as visitas à empresa verificou-se que existe um certo descompasso entre o que é efetivamente vendido e a expectativa do cliente; entre o que a área técnica e a área de suporte desenvolvem para a manutenção da operação. Esse descompasso tem gerado obstáculos e até sentimentos de incerteza nos relacionamentos pela dissonância de entendimento entre os agentes, ou seja, uma 
quebra entre o que o comercial vende, o que o departamento técnico desenvolve e aquilo que realmente o cliente necessita.

A carteira de clientes da empresa foi paulatinamente formada sem planejamento e sem um processo preestabelecido de prospecção. Para cada contrato firmado com um determinado cliente, a InfoLyn utiliza um de seus produtos de "prateleira" e realiza as customizações necessárias. Dessa forma, as customizações são recursos importantes da empresa no sentido de gerar argumento de vendas e valor agregado aos seus produtos.

No momento da venda as customizações são oferecidas para adequar o produto ao cliente, porém sempre existe um subdimensionamento das adaptações que serão necessárias, além disso, às atualizações dos sistemas que trazem novas necessidades que não foram contempladas na precificação, gerando novos custos não previstos.

Cada projeto possui especificidades e contingências não facilmente previstas, então essas customizações geralmente tomam mais tempo e recursos que o estimado para implantação e execução dos contratos, consequentemente o número de horas precificadas ex ante na elaboração do contrato é diferente daquele que a empresa utiliza para satisfazer o cliente, gerando adaptações contratuais e suporte técnico que oneram as operações com deslocamentos e tempo dedicados para transmitir conceitos fiscais e parametrizações que deveriam ser do conhecimento e do domínio dos contratantes, elevando os custos de transação em suas operações.

O desempenho na precificação de horas é considerado insatisfatório, pois constantemente é subdimensionado. O não envolvimento do usuário final no processo de desenvolvimento acarreta a necessidade de uma nova customização dos sistemas, além disso, a falta de integração entre as equipes comercial e de desenvolvimento impacta diretamente a satisfação do cliente e ao potencializar as dificuldades de operacionalização do sistema e a insatisfação do cliente, pode ocasionar quebra de contrato e gerar prejuízos.

$\mathrm{O}$ interesse próprio de cada área impacta tempestivamente na busca de soluções, assim como a falta de treinamento do pessoal de atendimento e suporte técnico nas novas atualizações e customizações aos clientes gera conflitos, ineficiência e insatisfação. Portanto, o problema parece não ser de capacidade técnica, mas de engajamento das áreas e integração da comunicação entre os colaboradores e os clientes. 
ADILSON ADERITO DA SILVA, ALBERTO DE MEDEIROS JÚNIOR, ANDREA CRISTINA DEIS RODRIGUES, ELISABETE DOS SANTOS FERNANDES, LUCAS AUGUSTO PEREZ DOS SANTOS, MARIANA PIRES DIAS

Ao longo dos últimos meses foram feitos mapeamentos das situações vivenciadas na empresa durante a execução de três contratos. Os itens identificados como a precificação, quebras de contrato, problemas de relacionamento e de integração e ruídos de comunicação que têm gerado custos e afetado a rentabilidade da empresa estão sintetizados no Quadro 1.

\begin{tabular}{|c|c|}
\hline Ex ante & Ex post \\
\hline $\begin{array}{l}\text { Racionalidade limitada na precificação de horas } \\
\text { - Contrato A - foram estimadas } 690 \text { horas para a } \\
\text { execução do projeto. }\end{array}$ & $\begin{array}{l}\text { Custo com adaptações e oportunismo } \\
\text { - Contrato A - Foram gastas } 1100 \text { horas frente às } 690 \text { horas } \\
\text { estimadas, perdas de R\$ } 94.050,00 \text { com adaptações contratuais } \\
\text { (valores a receber) }\end{array}$ \\
\hline $\begin{array}{l}\text { Racionalidade limitada e assimetria de informações no } \\
\text { diagnóstico de necessidades do cliente } \\
\text { - Contrato A - das } 690 \text { horas foram estimadas } 300 \\
\text { horas estimadas ao desenvolvimento do sistema com } \\
\text { Perdas de R\$ 67.650,00; }\end{array}$ & $\begin{array}{l}\text { Racionalidade limitada e assimetria de informações } \\
\text { - Contrato A. No total de } 1100 \text { horas, } 410 \text { podem ser classificadas } \\
\text { como retrabalho (conflito de informações). } \\
\text { Perdas com customizações não previstas em contrato. } \\
\text { - Cerca de } 70 \% \text { a } 80 \% \text { aceitam a repactuar o contrato. } \\
\text { Deslocamento e outros investimentos }\end{array}$ \\
\hline $\begin{array}{l}\text { Assimetria informações (falha coleta dados ex ante) } \\
\text { - Contrato B - Falha na coleta de informações com o } \\
\text { cliente no período de elaboração do contrato; } \\
\text { - Incerteza, informações incompletas ou desconhecidas, } \\
\text { tanto internas quanto externas. }\end{array}$ & $\begin{array}{l}\text { - Deslocamentos até o cliente, bonificações e treinamentos extras } \\
\text { em serviços sem cobranças; } \\
\text { - Gasto de horas adicionais com deslocamentos e refeição para } \\
\text { atendimento extra do cliente; } \\
\text { Falta ou atraso de suporte técnico } \\
\text { - Vários novos recursos tiveram que ser feitos; } \\
\text { - Recursos liberados com erros - retrabalho; } \\
\text { - Reclamações quanto ao tempo de atendimento; } \\
\text { - Gasto de } 20 \text { horas mensais com suporte comercial com custo } \\
\text { aproximado de R } \$ 2.200,00 \\
\text { - Rescisão contratual }\end{array}$ \\
\hline $\begin{array}{l}\text { Racionalidade limitada/ falta de comprometimento } \\
\text { - Contrato C - Acréscimo de serviço no módulo } \\
\text { negociado, com o compromisso de apoio do parceiro } \\
\text { para o desenvolvimento conjunto, durante a elaboração } \\
\text { do contrato. }\end{array}$ & $\begin{array}{l}\text { Oportunismo } \\
\text { - Contrato C - O cliente não deu o suporte prometido ao } \\
\text { desenvolvimento da solução. } \\
\text { - Quebra de contrato e prejuízo de R\$30.000,00. } \\
\text { - Aproveitamento de lacunas contratuais para cobrar entregas } \\
\text { maiores do que as previamente contratadas. } \\
\text { - Dispêndio de recursos para resolução conflitos com o cliente. } \\
\text { Consumo de } 10 \% \text { das horas destinadas ao desenvolvimento - } \\
\text { R\$ R\$ 7.200,00. }\end{array}$ \\
\hline
\end{tabular}

QUADRO 1 - Fontes de custo de transação ex ante e ex post

Fonte: Elaborado pelos autores; análise de três contratos com os clientes da empresa. 
A partir do panorama e diagnóstico apresentados, torna-se evidente a necessidade de implantação de mudanças nas práticas da InfoLyn, notadamente com relação a precificação de seus contratos, revisão de métodos e processo de comunicação entre os sócios, funcionários e clientes com vistas às melhorias nos índices de performance e satisfação dos clientes com melhorias de eficiência com a redução dos custos de transação.

\section{Custos de transação}

A redução dos custos de transação tem sido o foco dos estudos empreendidos por diversos autores de Organização Industrial desde a década de 1970 a partir da publicação dos trabalhos de Oliver Williamson que estruturaram a Teoria dos Custos de Transação (TCT). A TCT está fundamentada nos pressupostos comportamentais da racionalidade limitada e do comportamento oportunista dos atores nas negociações, a partir da premissa que, em sua essência, o ser humano tem a busca do próprio interesse como guia e, para isso, não hesitará em trapacear, mentir e até prejudicar o parceiro de negociação para obter alguma vantagem.
Tendo em vista a potencialidade das ações oportunistas nas negociações com os parceiros, Williamson (1979) defendeu a necessidade de se estabelecer contratos nas negociações como proteção contra o oportunismo, a fim de minimizar os custos de transação decorrentes de operações pelo mecanismo de preços do mercado, entendido como um sistema de procedimentos de negociação orientado pelos fortes incentivos que regula e legitima as trocas de produtos ou serviços entre agentes anônimos em cada transação.

Contudo, devido à racionalidade limitada do ser humano, não é possível elaborar contratos capazes de eliminar, por completo, os custos das ações oportunistas, pois há custos ex ante incorridos por conta do tempo dedicado à busca de informações relevantes para a elaboração dos contratos, escolha e negociação com parceiros de transação e, por outro lado, existem os custos ex post com as adaptações, renegociações e até quebra de contratos que surgem durante execução das atividades pactuadas entre os parceiros por conta de contingências não previstas na elaboração dos contratos, a exemplo do que foi diagnosticado no caso da InfoLyn,

Os custos de transação podem variar segundo a especificidade dos ativos tran- 
sacionados, a frequência com que as transações são realizadas com o parceiro e a incerteza de mercado que emerge de acontecimentos naturais ou comportamentais nas negociações. Portanto, a TCT defende que é possível minimizar esses custos e reduzir as perdas decorrentes de comportamentos oportunistas dos parceiros por meio da formalização de contratos em que são pactuados os comportamentos, as responsabilidades e as ações esperadas na transação criando-se um ambiente de negócios mais previsível.

Considerando o contexto de atuação da empresa InfoLyn e o seu estágio de desenvolvimento organizacional, fez-se o apontamento das atividades que a empresa desenvolve para implementar softwares voltados ao mercado de varejo que revelou potenciais fontes de custos de transação na área de suporte técnico da empresa. As informações coletadas nos bancos de dados dessa área foram analisadas com o suporte gráfico do software Tableau e valendo-se das métricas de desempenho da empresa fez-se uma análise de Risco, Custos, Benefícios e Oportunidades, processadas com o software de apoio à decisão Super Decisions, cujos processamentos apresentados a seguir culminaram em propostas de mu- danças para salvaguardar a empresa de ações oportunistas e reunir melhor nível de profissionalização das suas atividades.

\section{Visualização de dados}

A visualização de dados em forma de tabelas de planilhas eletrônicas e de gráficos (dashboards) é um recurso cada vez mais utilizado nas empresas para detectar novas oportunidades de negócios e possíveis falhas nos processos internos e externos no relacionamento com clientes. Os dashboards possibilitam a visualização de dados com interação com o usuário e, entre os mais aceitos pelo mercado, destacam-se o SAP Lumira, o Microsoft Power BI, o Tableau, o Cognos e o Qlick. Para esse diagnóstico, foi escolhido o Tableau, que é um sistema inicialmente desenvolvido na Universidade de Stanford que possibilita a visualização de dados para explorar e analisar bancos relacionais e cubos de dados.

Com a utilização do Tableau foi possível aprofundar o diagnóstico dos atendimentos realizados pelo Suporte Técnico da InfoLyn, avaliando-se as ligações realizadas, tempo de duração, operador, cliente e índice de satisfação. Para esta análise foram considerados os dados do trimestre 
composto pelos meses de janeiro, fevereiro e março, conforme a Figura 1, a seguir.

A partir do dashboard foi possível identificar-se com clareza as seguintes informações:

1. Quantidade de chamados por empresa, para identificar quais são os clientes que geram maior demanda à empresa (Figura 2).

2. Quantidade de chamados e satisfação por localidade, visando identificar a posição geográfica onde há mais insatisfação dos clientes (Figura 3).

\section{Chamados Por Mês}

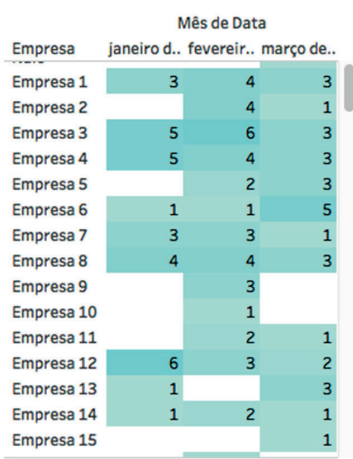

Chamados Por Operador

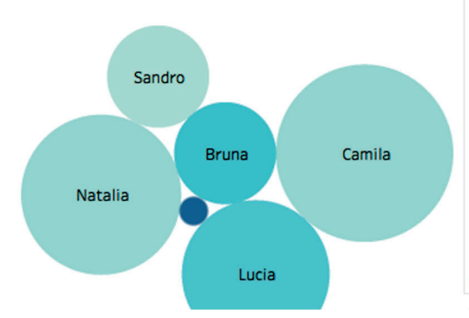

Média de Satisfação Por Estado

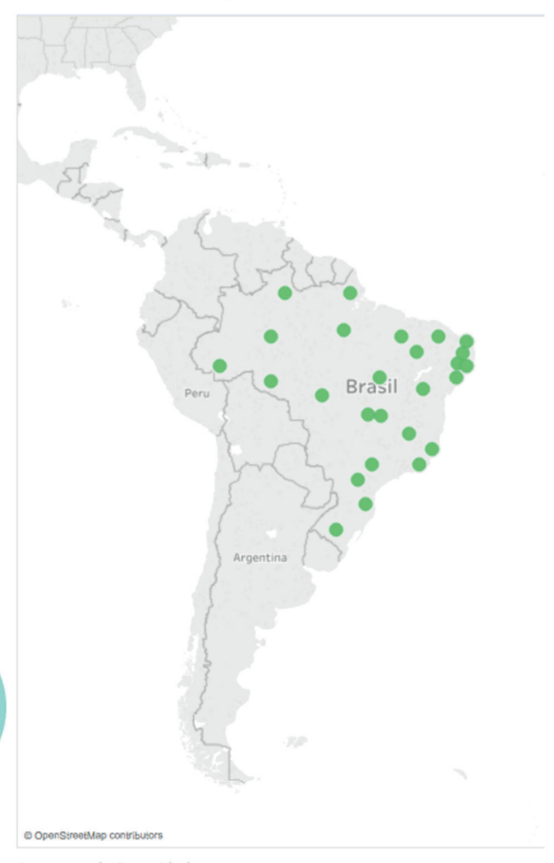

FIGURA 1 - Dashboard de satisfação de clientes da InfoLyn

Fonte: Dashboard elaborado pelos autores com dados da InfoLyn. Para interagir com o dashboard, acesse <http://infolyn.mack.adm.br $>$. 
ADILSON ADERITO DA SILVA, ALBERTO DE MEDEIROS JÚNIOR, ANDREA CRISTINA DEIS RODRIGUES, ELISABETE DOS SANTOS FERNANDES, LUCAS AUGUSTO PEREZ DOS SANTOS, MARIANA PIRES DIAS

\section{Quantidade de Chamados por Empresa}

\begin{tabular}{lll}
\hline Empresa & Localidade & \\
\hline Nulo & Santa Catarina & \\
\hline Empresa 1 & Santa Catarina & \\
\hline Empresa 2 & Ceará & \\
\hline Empresa 3 & São Paulo & \\
\hline Empresa 4 & Rio Grande do Sul & \\
\hline Empresa 5 & São Paulo & \\
\hline Empresa 6 & São Paulo & \\
\hline Empresa 7 & São Paulo & \\
\hline Empresa 8 & São Paulo & \\
\hline Empresa 9 & São Paulo & \\
\hline Empresa 10 & Sergipe & \\
\hline Empresa 11 & Distrito Federal & \\
\hline Empresa 12 & São Paulo & \\
\hline Empresa 13 & Tocantins & \\
\hline Empresa 14 & Mato Grosso & \\
\hline Empresa 15 & Amapá & \\
\hline Empresa 16 & São Paulo & \\
\hline Empresa 17 & Roraima & \\
\hline Empresa 18 & Alagoas \\
\hline Empresa 19 & São Paulo & \\
\hline Empresa 20 & São Paulo & \\
\hline Empresa 21 & Paraiba \\
\hline Empresa 22 & Pará \\
\hline Empresa 23 & Sergipe \\
\hline Empresa 24 & Rio Grande do Sul & \\
\hline \hline
\end{tabular}

FigurA 2 - Gráfico filtrado da demanda de chamadas dos clientes da InfoLyn Fonte: Dashboard elaborado pelos autores com dados da InfoLyn.

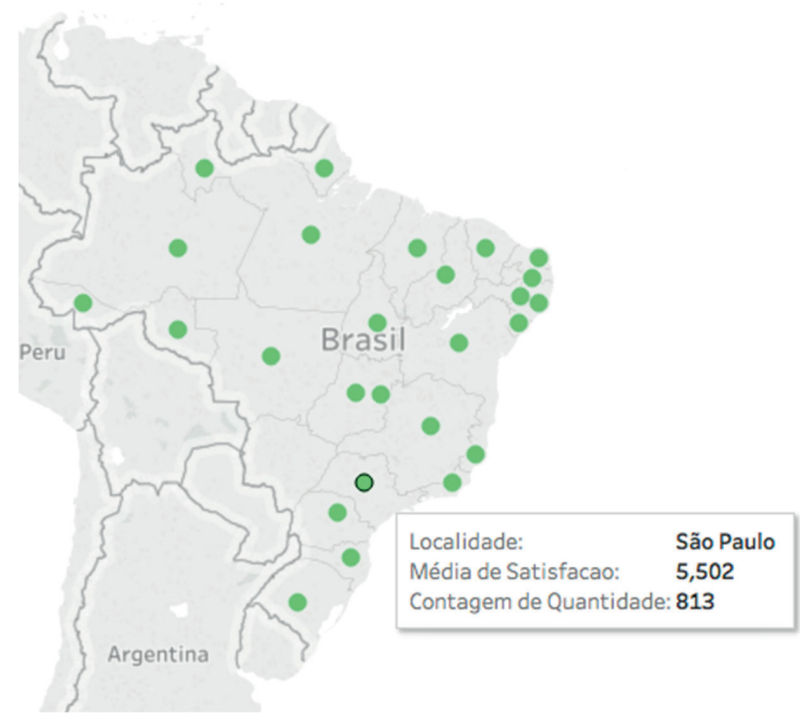

FIGURA 3 - Distribuição geográfica dos clientes insatisfeitos da InfoLyn Fonte: Dashboard elaborado pelos autores com dados da InfoLyn. 
3. Duração total (min) dos chamados, para identificar quais são os clientes com chamadas mais demoradas (Figura 4).

4. Índice de principais problemas (Figura 5).

5. Identificação de quais operadores são mais demandados (FIgura 6).

\section{Sistema de Apoio à Decisão}

Diante do diagnóstico apresentado no Tableau, reuniram-se as principais informações para estabelecer um racional de atuação edefinir as possibilidades de implantação de melhorias. Para tanto, as análises foram subdivididas e avaliadas sob os aspectos de benefícios, oportunidades, custos e riscos, utilizando-se o Sistema de Apoio à Decisão (SAD) SuperDecisions.

O SuperDecisions é baseado no Analytical Network Process (ANP), que é um modelo matemático desenvolvido pelo Prof. Thomas Saaty da Universidade de Pittsburgh que possibilita, por meio de comparações par a par entre os fatores de decisão, que se encontre uma solução boa o suficiente para se resolver um problema de administração.

Na comparação par a par entre os fatores de decisão são atribuídas notas, conforme a escala fundamental (Quadro 2).

\begin{tabular}{lrr|} 
Duracão Total dos Chamados \\
Empresa & Contag.. & Duraca.. \\
\hline Empresa 1 & 10 & 1.863 \\
Empresa 2 & 5 & 1.482 \\
Empresa 3 & 14 & 3.500 \\
Empresa 4 & 12 & 2.955 \\
Empresa 5 & 5 & 1.041 \\
Empresa 6 & 7 & 1.996 \\
Empresa 7 & 7 & 958 \\
Empresa 8 & 11 & 1.985 \\
Empresa 9 & 3 & 611 \\
Empresa 10 & 1 & 144 \\
Empresa 11 & 3 & 643 \\
Empresa 12 & 11 & 2.241 \\
Empresa 13 & 4 & 1.152 \\
Empresa 14 & 4 & 371 \\
Empresa 15 & 1 & 294 \\
\hline
\end{tabular}

FIGURA 4 - Duração das chamadas por cliente da InfoLyn Fonte: Dashboard elaborado pelos autores com dados da InfoLyn.

\section{Índice de Problemas}

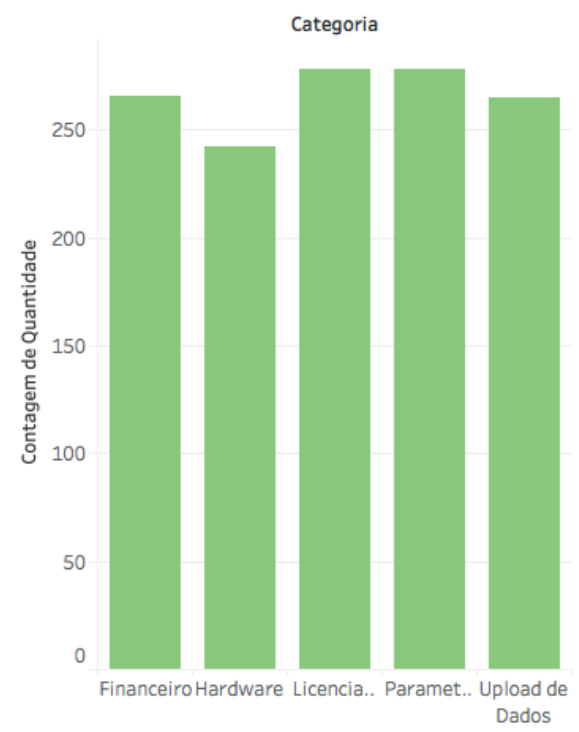

FIGURA 5 - Identificação dos principais problemas dos clientes da InfoLyn

Fonte: Dashboard elaborado pelos autores com dados da InfoLyn. 
Saaty (2005) sugere que a análise de

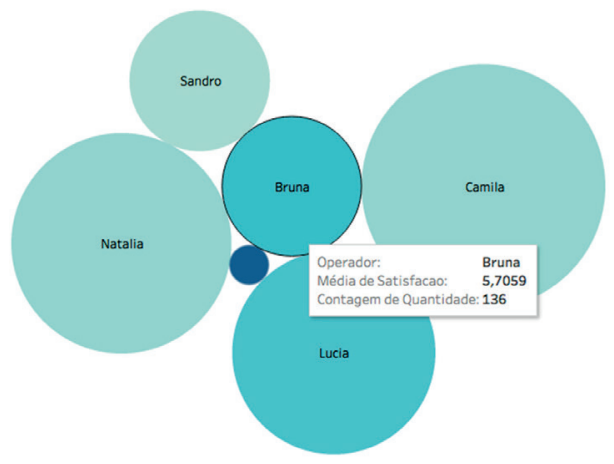

FIGURA 6 - Identificação dos operadores da InfoLyn Fonte: Dashboard elaborado pelos autores com dados da Infolyn. um problema pode ser feita a partir de quatro méritos, como ele os denomina: Benefícios, Oportunidades, Custos e Riscos.

Essas relações são chamadas por ele de méritos BOCR (Benefits, Opportunities, Costs and Risks), em que os méritos de benefícios (B) e oportunidades (O) medem as contribuições ou importâncias positivas, enquanto os méritos de custo (C) e risco $(\mathrm{R})$ medem as prioridades negativas, se elas existirem (SAATY, 2005).

\begin{tabular}{|c|c|c|}
\hline Intensidade & Definição & Explicação \\
\hline 1 & Mesma importância & As duas atividades contribuem igualmente para o objetivo. \\
\hline 2 & Fraca importância & Entre igual e moderada importância. \\
\hline 3 & Importância moderada & $\begin{array}{l}\text { A experiência ou julgamento é fracamente a favor de uma } \\
\text { atividade sobre outra. }\end{array}$ \\
\hline 4 & Moderada para forte importância & Entre moderada e forte importância. \\
\hline 5 & Fortemente importante & $\begin{array}{l}\text { A experiência ou julgamento é fortemente a favor de uma } \\
\text { atividade sobre outra. }\end{array}$ \\
\hline 6 & $\begin{array}{l}\text { Fortemente para muito fortemente } \\
\text { preferível }\end{array}$ & Entre forte e muito forte importância. \\
\hline 7 & $\begin{array}{l}\text { Muito fortemente ou demonstra } \\
\text { importância }\end{array}$ & $\begin{array}{l}\text { Uma atividade é muito fortemente preferida sobre outra. A } \\
\text { sua dominância é possível na prática. }\end{array}$ \\
\hline 8 & Importância quase extrema & Entre muito forte e extrema importância. \\
\hline 9 & Extrema importância & $\begin{array}{l}\text { A evidência de preferência de uma atividade pode ser } \\
\text { afirmada em sua mais elevada possibilidade. }\end{array}$ \\
\hline $\begin{array}{l}\text { Valores } \\
\text { recíprocos }\end{array}$ & \multicolumn{2}{|c|}{$\begin{array}{l}\text { Se uma primeira atividade comparada a uma segunda atividade tem valor igual a um dos valores de } \\
\text { intensidade indicados acima, então esta segunda atividade tem o valor de intensidade recíproco à primeira. }\end{array}$} \\
\hline
\end{tabular}

QUADRO 2 - Escala fundamental do ANP

Fonte: Saaty $(2000,2001,2005)$. 
O Prof. Saaty além de ter desenvolvido o modelo matemático teórico, também supervisionou o desenvolvimento do SuperDecisions,

Os fatores de decisão utilizados no estudo foram

- Benefícios: com o objetivo de obter ganhos em escala, do ponto de vista financeiro, técnico e humano, os critérios avaliados foram: "eficiência", "qualificação da equipe" e "satisfação dos clientes".

- Oportunidades: com o objetivo de identificar e aproveitar oportunidades, a partir da ampliação e melhoria na atuação, definiram-se como critérios de oportunidades o "aumento de receitas", a "aproximação com o cliente" (relacionamento), a "possibilidade de prospecção de novos clientes" e a "retenção de talentos".

- Custos: diante do objetivo de otimizar recursos, a partir das análises em questão, foram definidos como critérios de avaliação os custos envolvidos com a “capacitação de funcionários", "abertura de escritórios regionalizados", "custos de transação" e de "novas contratações”.

- Riscos: do ponto de vista da avaliação de riscos, com o objetivo de identificar os principais impactos e preveni-los, foram selecionados os critérios de "dis- ponibilidade do operador”, “elevação do custo financeiro" e "qualidade do atendimento".

O Suporte Técnico foi considerado como o setor da empresa selecionado para o presente trabalho, pois está diretamente ligado à satisfação dos clientes e consequentemente à manutenção e perenidade da empresa no mercado. Trata-se de setor estratégico da InfoLyn, pois pode apoiar como protagonista no relacionamento com os clientes, atrair novos contratos e diminuir custos de diversas origens, como os de transação.

No racional de atuação proposto, foram selecionadas três novas possibilidades de implementação de melhorias, além da possibilidade de manutenção do atual modelo:

1. Manutenção da Centralização: esta opção não traz mudanças à atual forma de atuação do Suporte Técnico, ou seja, os clientes são atendidos conforme suas ligações, pelo atendente que estiver disponível.

2. Manutenção da Centralização com Capacitação: nesta opção, os atendentes receberiam tratamento voltado para o atendimento ao cliente, com foco na satisfação e resolução de problemas apresentados. 
3. Regionalização de Suporte: esta opção visa aproximar o Suporte Técnico dos principais clientes, sob forma de descentralização e maior especialização do atendimento, com possibilidade de visitas técnicas e outras complementariedades ao atendimento.

4. Segmentação - Gerenciamento de Contas: esta opção tem como objetivo especializar o atendimento conforme importância e necessidades do cliente, trazendo a figura do Gerente de Contas, ou seja, cada cliente já saberá quem é o atendente do suporte responsável pelo apoio à sua empresa.

Baseando-se nas informações inseridas e disponibilizadas pelo SuperDecisions, foi possível comparar as opções sob os diversos aspectos.

O diagrama apresentado na Figura 7 representa a relação entre o objetivo geral de elevação da satisfação dos clientes internos e externos da InfoLyn consideradas as sub-redes dos méritos BOCR.

Os fatores de decisão para cada mérito (sub-rede) foram selecionados pelos consultores do projeto.

A. Avaliações da sub-rede do mérito Benefícios

Para a sub-rede do mérito Benefícios, foram considerados os fatores de decisão, conforme apresentados no Quadro 3.

Para a obtenção do resultado para o objetivo visando a elevação da satisfação dos clientes internos e externos da empresa no mérito Benefícios, inicialmente os fatores de decisão foram comparados par a par e atribuídas notas, conforme escala apresentada no Quadro 1 e, depois, para cada fator de decisão, as opções para a elevação da satisfação foram comparadas par a par utilizando-se as notas pelos mesmos critérios.

Objetivo geral

Elevação da satisfação dos clientes internos e externos

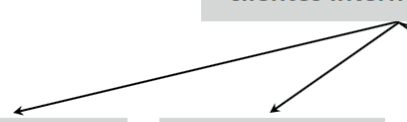

Sub-rede Benefícios
Sub-rede Oportunidades

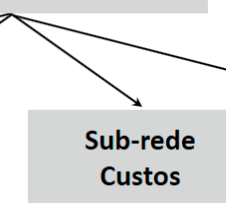

Sub-rede

Riscos

FIGURA 7 - Diagrama para a elevação da satisfação dos clientes internos e externos da InfoLyn Fonte: Elaborado pelos autores. 
INFOLYN - ELEVANDO A SATISFAÇÃO DOS CLIENTES INTERNOS E EXTERNOS

\begin{tabular}{ll}
\hline Fatores de decisão & Explicação \\
\hline Eficiência & Mensuração do comportamento dos índices de eficiência. \\
\hline Qualificação da equipe & Mensuração do comportamento dos níveis de qualificação da equipe. \\
\hline Satisfação do cliente & Mensuração do comportamento dos níveis de satisfação dos clientes. \\
\hline
\end{tabular}

QUADRO 3 - Fatores de decisão considerados para o mérito benefícios

Fonte: Elaborado pelos autores.

O diagrama apresentado na Figura 8 representa essas comparações.

Como exemplo, para a sub-rede do mérito Benefícios, foram atribuídas notas conforme escala apresentada no Quadro 2, seguindo-se as etapas:

1. Os fatores de decisão foram comparados par a par.
O fator "Eficiência" foi considerado moderadamente mais importante do que o fator "Qualificação da equipe". "Satisfação do cliente" foi considerado moderadamente mais importante do que o fator "Eficiência" e fortemente mais importante que "Eficiência" (Figura 9).

Mérito

Fatores de decisão

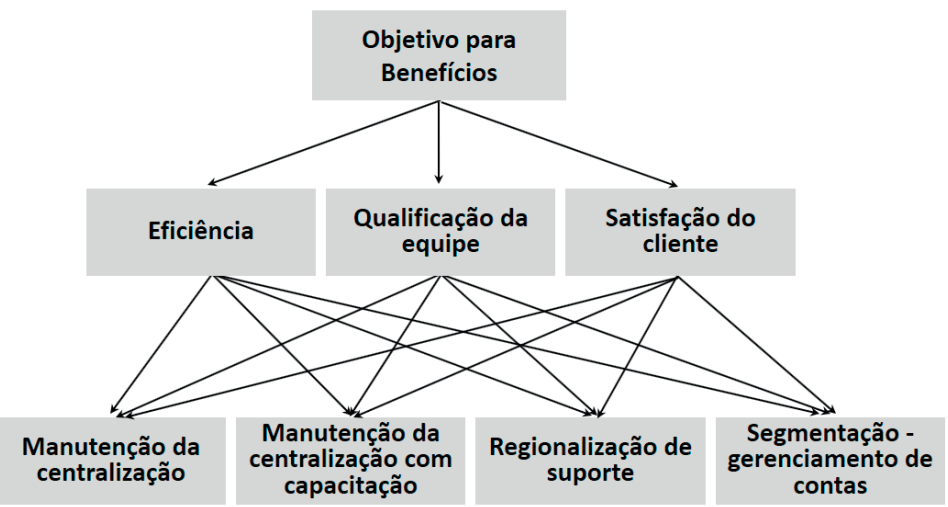

FIGURA 8 - Diagrama para a elevação da satisfação dos clientes internos e externos no mérito benefícios

Fonte: Elaborado pelos autores. 
ADILSON ADERITO DA SILVA, ALBERTO DE MEDEIROS JÚNIOR, ANDREA CRISTINA DEIS RODRIGUES, ELISABETE DOS SANTOS FERNANDES, LUCAS AUGUSTO PEREZ DOS SANTOS, MARIANA PIRES DIAS

\begin{tabular}{|c|c|c|c|c|c|c|c|c|c|c|c|c|c|c|c|c|c|c|c|c|c|}
\hline 1. & Eficiência & $>=9.5$ & 9 & 8 & 7 & 6 & 5 & 4 & 3 & 2 & 2 & 3 & 4 & 5 & 6 & 7 & 8 & 9 & $>=9.5$ & No comp. & Qualificação \\
\hline 2. & Eficiência & $>=9.5$ & 9 & 8 & 7 & 6 & 5 & 4 & 3 & 2 & 2 & 3 & 4 & 5 & 6 & 7 & 8 & 9 & $>=9.5$ & No comp. & Satisfação d \\
\hline & alificação & $>=9.5$ & 9 & 8 & 7 & 6 & 5 & 4 & 3 & 2 & 2 & 3 & 4 & 5 & 6 & 7 & 8 & 9 & $>=9.5$ & No comp. & Satisfação d \\
\hline
\end{tabular}

FIgURA 9 - Tela de julgamento dos fatores de decisão para mérito Benefícios Fonte: Elaborada pelos autores.

2. As opções da satisfação dos clientes internos e externos de cada fator de decisão de Benefícios foram comparadas par a par.

Tomando-se, por exemplo, por base, $\mathrm{o}$ fator de decisão "Eficiência" os julgamentos foram feitos conforme a Figura 10.

Com as notas atribuídas, para o fator "Eficiência" os resultados foram, em ordem de preferência:
- Segmentação - gerenciamento de contas: $55,5 \%$

- Regionalização de suporte: $23,8 \%$

- Manutenção da centralização com capacitação: $15,3 \%$

- Manutenção da centralização: 5,4\%

A segunda etapa deve ser repetida tomando-se por base os demais fatores de decisão: "Qualificação da equipe"; e "Satisfação do cliente”.

\begin{tabular}{|c|c|c|c|c|c|c|c|c|c|c|c|c|c|c|c|c|c|c|c|c|}
\hline 1. Central/Capa & $>=9.5$ & 9 & 8 & 7 & 6 & 5 & 4 & 3 & 2 & 2 & 3 & 4 & 5 & 6 & 7 & 8 & 9 & $>=9.5$ & No comp. & Centralizaçã \\
\hline 2. Central/Capa & $>=9.5$ & 9 & 8 & 7 & 6 & 5 & 4 & 3 & 2 & 2 & 3 & 4 & 5 & 6 & 7 & 8 & 9 & $>=9.5$ & No comp. & Regionalizaç \\
\hline 3. Central/Capa & $>=9.5$ & 9 & 8 & 7 & 6 & 5 & 4 & 3 & 2 & 2 & 3 & 4 & 5 & 6 & 7 & 8 & 9 & $>=9.5$ & No comp. & Segmentação: \\
\hline 4. Centralizaçã & $>=9.5$ & 9 & 8 & 7 & 6 & 5 & 4 & 3 & 2 & 2 & 3 & 4 & 5 & 6 & 7 & 8 & 9 & $>=9.5$ & No comp. & Regionalizaç \\
\hline 5. Centralizaçã & $>=9.5$ & 9 & 8 & 7 & 6 & 5 & 4 & 3 & 2 & 2 & 3 & 4 & 5 & 6 & 7 & 8 & 9 & $>=9.5$ & No comp. & Segmentaçäo: \\
\hline 6. Regionalizaç $\sim$ & $x=9.5$ & 9 & 8 & 7 & 6 & 5 & 4 & 3 & 2 & 2 & 3 & 4 & 5 & 6 & 7 & 8 & 9 & $>=9.5$ & No comp. & Segmentação: \\
\hline
\end{tabular}

Figura 10 - Tela de julgamento das opções para "Eficiência" Fonte: Elaborada pelos autores. 
Os resultados das preferências pelas opções da elevação da satisfação dos clientes internos e externos para o mérito Benefícios foram, em ordem de preferência:

- Segmentação - gerenciamento de contas: $56,4 \%$

- Regionalização de suporte: $22,4 \%$

- Manutenção da centralização com capacitação: $15,2 \%$

- Manutenção da centralização: 6,0\%

B. Avaliações da sub-rede do mérito Oportunidades

Para a sub-rede do mérito Oportunidades, foram considerados os fatores de decisão, conforme apresentados no Quadro 4.

Para a obtenção do resultado para o objetivo para a elevação da satisfação dos clientes internos e externos no mérito Oportunidades, inicialmente os fatores de decisão foram comparados par a par e atribuídas notas, conforme escala apresentada no Quadro 2 e, depois, para cada fator de decisão, as opções para a elevação da satisfação dos clientes internos e externos foram comparadas par a par utilizando-se as notas pelos mesmos critérios. O diagrama apresentado na Figura 11 representa essas comparações.

As comparações par a par seguem as mesmas etapas da sub-rede do mérito Oportunidades:

1. Os fatores de decisão "Elevação de receita", "Prospecção de novos clientes", "Relacionamento com clientes", "Retenção de talentos" foram comparados par a par.

2. As opções da satisfação dos clientes internos e externos de cada fator de decisão de Oportunidades foram comparadas par a par.

Esta etapa deve ser aplicada na comparação par a par das opções "Manutenção

\begin{tabular}{ll}
\hline Fatores de decisão & Explicação \\
\hline Elevação de receita & A partir das melhorias esperadas aumentar os valores de receita auferidos. \\
\hline Prospecção de novos clientes & A partir das melhorias esperadas ampliar a carteira de clientes. \\
\hline Relacionamento com cliente & $\begin{array}{l}\text { A partir das melhorias esperadas gerar maior proximidade aos clientes } \\
\text { atendidos. }\end{array}$ \\
\hline Retenção de talentos & A partir das melhorias esperadas identificar e reter talentos. \\
\hline
\end{tabular}

QUADRO 4 - Fatores de decisão considerados para o mérito Oportunidades Fonte: Elaborado pelos autores. 


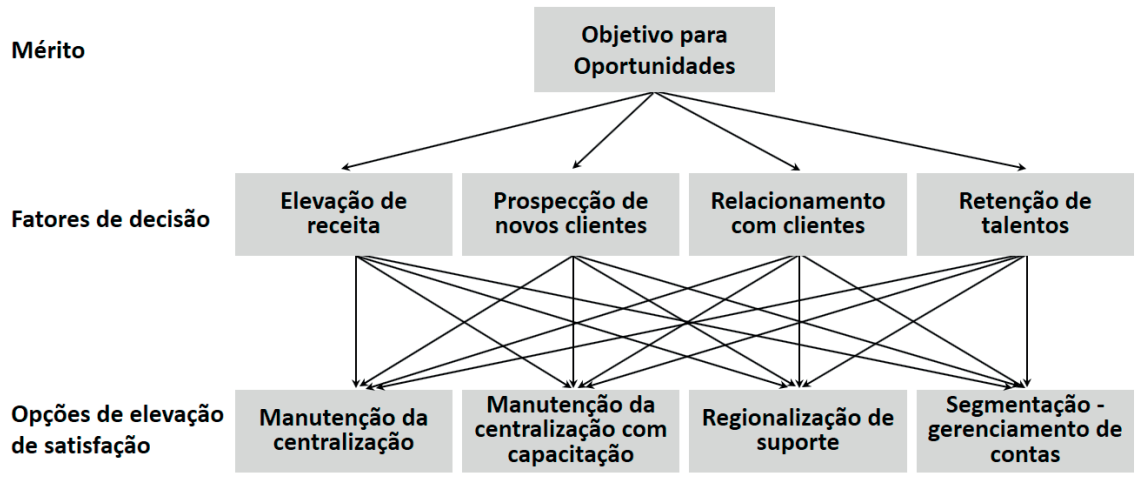

FIGURA 11 - Diagrama para a elevação da satisfação dos clientes internos e externos no mérito Oportunidades

Fonte: Elaborado pelos autores.

da Centralização", "Manutenção da Centralização com capacitação", "Regionalização de Suporte" e "Segmentação - Gerenciamento de Contas", tomando-se por base cada um dos fatores de decisão.

Os resultados das preferências pelas opções da elevação da satisfação dos clientes internos e externos para o mérito Oportunidades foram, por ordem de preferência:

- Segmentação - gerenciamento de contas: $55,9 \%$

- Regionalização de suporte: $26,0 \%$

- Manutenção da centralização com capacitação: $13,4 \%$

- Manutenção da centralização: 4,7\% c. Avaliações da sub-rede do mérito Custos

Para a sub-rede do mérito Custos, foram considerados os fatores de decisão, conforme apresentados no Quadro 5.

Para a obtenção do resultado para o objetivo para a elevação da satisfação dos clientes internos e externos no mérito Custos, inicialmente os fatores de decisão foram comparados par a par e atribuídas notas, conforme escala apresentada no Quadro 1 e, depois, para cada fator de decisão, as opções de elevação de satisfação foram comparadas par a par utilizando-se as notas pelos mesmos critérios.

O diagrama apresentado na Figura 12 representa essas comparações. 
INFOLYN - ELEVANDO A SATISFAÇÃO DOS CLIENTES INTERNOS E EXTERNOS

\begin{tabular}{ll}
\hline Fatores de decisão & Explicação \\
\hline Capacitação & Valores envolvidos nos investimentos em capacitação de funcionários. \\
\hline Contratação & Valores envolvidos nos investimentos para contratação de novos colaboradores. \\
\hline Escritório regional & Valores envolvidos nos investimentos para criação de escritórios regionais. \\
\hline Transação & Valores envolvidos nos contratos firmados ex ante e ex post. \\
\hline
\end{tabular}

QUADRO 5 - Fatores de decisão considerados para o mérito Custos

Fonte: Elaborado pelos autores.

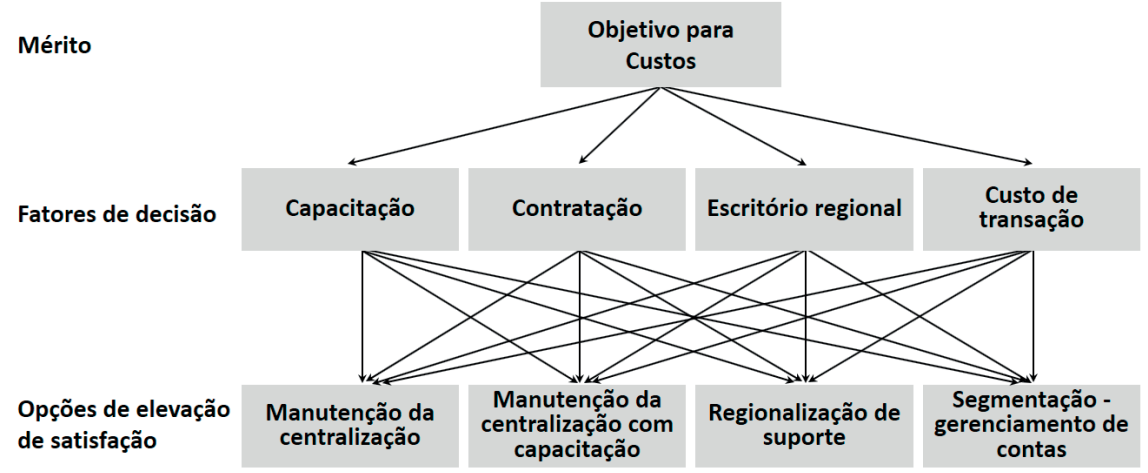

FIGURA 12 - Diagrama para a elevação da satisfação dos clientes internos e externos no mérito Custos Fonte: Elaborado pelos autores.

As comparações par a par seguem as mesmas etapas da sub-rede do mérito Custos:

1. Os fatores de decisão "Capacitação", "Contratação", "Escritório regional", “Custo de transação” foram comparados par a par.

2. As opções da satisfação dos clientes internos e externos de cada fator de decisão de Custos foram comparadas par a par.
Essa etapa deve ser aplicada na comparação par a par das opções "Manutenção da Centralização", "Manutenção da Centralização com capacitação", "Regionalização de Suporte" e "Segmentação - Gerenciamento de Contas” tomando-se por base cada um dos fatores de decisão. 
Os resultados das preferências pelas opções da elevação da satisfação dos clientes internos e externos para o mérito Custos foram, por ordem de preferência:

- Manutenção da centralização: $41,8 \%$

- Manutenção da centralização com capacitação: $31,2 \%$

- Segmentação - gerenciamento de contas: $17,2 \%$

- Regionalização de suporte: 9,8\%

D. Avaliações da sub-rede do mérito Riscos

Para a sub-rede do mérito Riscos, foram considerados os fatores de decisão, conforme apresentados no Quadro 6.

Para a obtenção do resultado para o objetivo para a elevação da satisfação dos clientes internos e externos no mérito Riscos, inicialmente os fatores de decisão foram comparados par a par e atribuídas notas, conforme escala apresentada no Quadro 1 e, depois, para cada fator de decisão, as opções de elevação de satisfação foram comparadas par a par utilizando-se as notas pelos mesmos critérios.

O diagrama apresentado na Figura 13 representa essas comparações.

As comparações par a par seguem as mesmas etapas da sub-rede do mérito Riscos:

1. Os fatores de decisão "Disponibilidade do operador", "Elevação de custo/benefícios financeiros" e "Qualidade de atendimento" foram comparados par a par".

2. As opções da satisfação dos clientes internos e externos de cada fator de decisão de Riscos foram comparadas par a par.

Essa etapa deve ser aplicada na comparação par a par das opções "Manutenção da Centralização", "Manutenção da Centralização com capacitação", "Regionalização de Suporte" e "Segmentação Gerenciamento de Contas", tomando-se por base cada um dos fatores de decisão.

\begin{tabular}{ll}
\hline Fatores de decisão & Explicação \\
\hline Disponibilidade do operador & Disponibilidade de atendimento tempestivo e resolutivo. \\
\hline Elevação custo/benefícios financeiros & Avaliação da relação custo/benefícios dos investimentos. \\
\hline Qualidade de atendimento & Qualidade no atendimento prestado pelos operadores. \\
\hline
\end{tabular}

QUADRO 6 - Fatores de decisão considerados para o mérito Riscos Fonte: Elaborado pelos autores. 


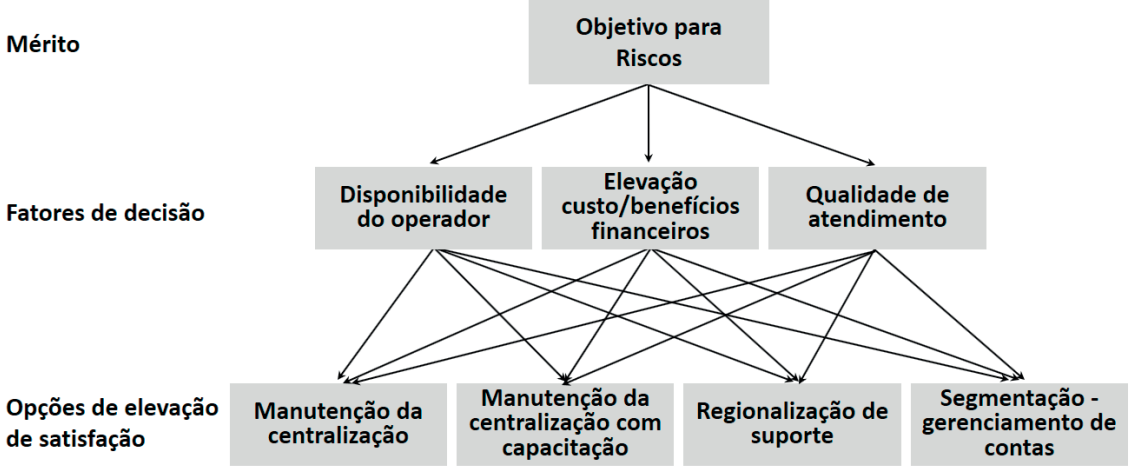

FIGURA 13 - Diagrama para a elevação da satisfação dos clientes internos e externos no mérito Riscos Fonte: Elaborado pelos autores.

Os resultados das preferências pelas opções da elevação da satisfação dos clientes internos e externos para o mérito Riscos foram, por ordem de preferência:

- Segmentação - gerenciamento de contas: $51,1 \%$

- Regionalização de suporte: $18,7 \%$

- Manutenção da centralização com capacitação: $19,7 \%$

- Manutenção da centralização: 10,5\%
E. Avaliação final

A Tabela 1 sintetiza os resultados das preferências pelas opções de elevação de satisfação de cada mérito.

Observa-se, pelos resultados para cada mérito, uma forte dispersão de preferências entre os países. $\mathrm{O}$ fator "Segmentação - gerenciamento de contas" destacou-se frente aos demais nos quesitos de Benefícios, Oportunidades e Riscos. Já na

TABELA 1 - Síntese dos resultados das preferências pelas opções de elevação de satisfação

\begin{tabular}{lcccc}
\hline & Benefícios & Oportunidades & Custos & Riscos \\
\hline Segmentação - gerenciamento de contas & $56,4 \%$ & $55,9 \%$ & $17,2 \%$ & $51,1 \%$ \\
\hline Manutenção da centralização com capacitação & $15,2 \%$ & $13,4 \%$ & $31,2 \%$ & $19,7 \%$ \\
\hline Manutenção da centralização & $6,0 \%$ & $4,7 \%$ & $41,8 \%$ & $10,5 \%$ \\
\hline Regionalização de suporte & $22,4 \%$ & $26,0 \%$ & $9,8 \%$ & $18,7 \%$ \\
\hline
\end{tabular}

Fonte: Elaborada pelos autores. 
avaliação de Custos, a Manutenção da centralização apresentou o melhor resultado.

Ao final dos julgamentos par a par dos 14 fatores de decisão na síntese dos resultados encontrados na avaliação dos méritos BOCR observa-se, pela coluna "Normals" da Figura 14, que houve preferência pela opção "Segmentação - gerenciamento de contas", com 38,4\%. As opções "Manutenção da centralização com capacitação" e "Manutenção da centralização" praticamente empatam no segundo lugar da preferência, com respectivamente $22,0 \%$ e 21,8\% e a opção "Regionalização do suporte" é a menos preferida, com 17,8\% para a elevação da satisfação dos clientes internos e externos.

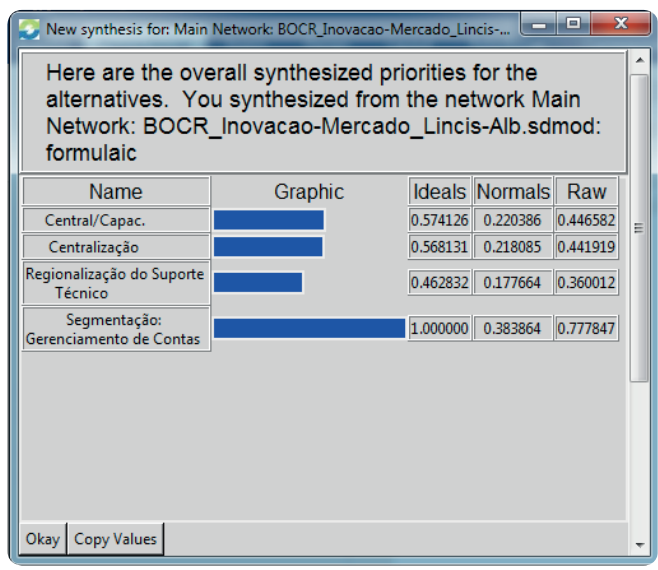

FIgURA 14 - Resultado final Fonte: Elaborada pelos autores.

\section{CONSIDERAÇÕES FINAIS}

O objetivo deste relato consistiu em apresentar um diagnóstico relacionado a área de suporte técnico da InfoLyn e, com base neste, identificar por Sistema de Apoio à Decisão a solução boa o suficiente a ser adotada levando em consideração a melhoria da eficiência da área com a redução dos riscos e custos de transação.

A empresa depara hodiernamente com a necessidade de gerenciar situações de incerteza, provocadas por assimetrias de informações entre as partes, tanto interna quanto externamente. Essa assimetria de informações, potencializada pela racionalidade limitada dos agentes, evidenciou que a necessidade do cliente é, algumas vezes, entendida de forma dissonante do serviço que a área técnica desenvolve e dá suporte para manutenção da operação daquilo que efetivamente foi vendido.

Destaca-se ainda que o usuário final só conhece o produto pronto no ato da implantação, o que traz à InfoLyn impactos negativos e custos de transação ex post, pois a ausência de conhecimento do produto final, a priori, dificulta a sua utilização pelos usuários, eleva o número de customizações necessárias às operações e onera a área de 
Suporte Técnico e de desenvolvimento da empresa com custos de transação ex post.

A assimetria de informações também foi evidenciada no relacionamento entre as áreas internas da empresa que são lideradas individualmente pelos sócios. Esse tipo de atuação, além de gerar assimetrias de informações internas, tem limitado o progresso técnico e gerado dificuldades comportamentais como disputas por poder e recursos e a busca de interesses próprios de cada área, culminando em conflitos, desvio de foco e prejuízo aos objetivos organizacionais comuns da empresa.

A análise dos dados de suporte pelo dashboard Tableau possibilitou constatar que a base de clientes está dividida entre os estados brasileiros, aumentando os eventuais custos de regionalização, enquanto que o índice de chamados encerrados com sucesso é muito maior que os não encerrados, dessa forma a técnica remota mostra-se eficiente. Nota-se, porém, que o nível de profissionalismo da equipe precisa melhorar, um vez que ainda é expressivo o índice de não resolução. Exemplo disso é um número elevado de necessidade de suporte no quesito Financeiro e Licenciamento, assuntos esses que fogem em uma primeira análise da relação com problemas técnicos, devendo a InfoLyn estabelecer uma nova estratégia para estes chamados.

Não existe isonomia na distribuição de chamados, nem nível de expertise dos operadores para cada determinado assunto e os chamados para um mesmo assunto ou dúvida possuem tempo de atendimento muito divergente, demonstrando falta de preparo nos operadores para lidar com temas cotidianos. Dessa forma, em uma abordagem valendo-se de métricas já definidas, com o auxílio do software SuperDecisions, em uma análise de Benefícios, Oportunidades, Custo e Risco, a segmentação e a criação do cargo de gerente de contas torna-se vantajosa, uma vez que garante a isonomia do atendimento, profissionaliza o atendente e economiza eventuais custos de transação contemplados na regionalização.

Outro aspecto que chamou a atenção nas operações da empresa foi a incerteza e a imprecisão quanto à precificação do tempo estimado para a execução dos serviços contratados, fato que carece mais atenção e assertividade da empresa, uma vez que geram desgastes, conflitos e perdas com retrabalho decorrentes das adaptações necessárias na customização dos produtos aos clientes que nem sempre são compensadas financeiramente em sua totalidade, 
gerando prejuízos financeiros, custos de transação e perda de eficiência operacional e de desempenho.

\section{INFOLYN - RAISING THE SATISFACTION OF INTERNAL AND EXTERNAL CUSTOMERS}

\section{ABSTRACT}

InfoLyn develops and implements software aimed at the retail market throughout the national territory. In light of the assumptions and dimensions of Transaction Costs Theory (TCT), in this report, the results are presented observations that culminated in the mapping the primary sources of costs that emerge in the Technical Support Area of the company. However, the limited rationality of agents enhances the asymmetry of information in InfoLyn and gives scope for opportunistic behavior, problems of team management, service of the customer base and, consequently, transaction costs. We observed that users have difficulty in using the systems and that the high number of customizations necessary for the operations burdens the Technical Support Area. As a practical result of the study, we proposed to the company alternatives based on processing information to improve the area of technical support decision-making databases software. These alternatives will allow InfoLyn act as a protagonist in the relationship with customers, attract new contracts, and reduce the transaction and operational costs identified.

\section{KEYWORDS}

Transaction Costs. Technical Support. Decision Support. Integration.

\section{REFERÊNCIAS}

ABES - Associação Brasileira das Empresas de Software. Mercado Brasileiro de Software Panorama e Tendências. 2016. Disponível em: <http://central.abessoftware.com.br/ Content/UploadedFiles/Arquivos/Dados \%202011/ABES-Publicacao-Mercado2016.pdf>. Acesso em: 20 maio 2017.

SAATY, T. L. Fundamentals of decision making and priority theory: with the Analytic Hierarchy Process. Pittsburgh: RWS Publications, 2000.

SAATY, T. L. Decision making with dependence and feedback. The analytic network process. 2. ed. Pittsburgh: RWS Publications, 2001.

SAATY, T. L. Theory and applications of the analytic network process. Decision making with benefits, opportunities, costs, and risks. Pittsburgh: RWS Publications, 2005.

WILLIAMSON, O. E. Transaction-Cost Economics: The Governance of Contractual. Journal of Law and Economics, v. 22, n. 2, p. 233-261, 1979. 